\title{
Automated 3D immunofluorescence analysis of Dorsal Root Ganglia for the investigation of neural circuit alterations: a preliminary study.
}

\author{
Santa Di Cataldo, Simone Tonti, \\ Enrico Macii, Elisa Ficarra \\ Dipartimento di Automatica e Informatica \\ Politecnico di Torino \\ Corso Duca degli Abruzzi 24, 10129 Torino (Italy) \\ Email: santa.dicataldo, simone.tonti@polito.it \\ enrico.macii, elisa.ficarra@polito.it
}

\author{
Elisa Ciglieri, Francesco Ferrini, \\ Chiara Salio \\ Dipartimento di Scienze Veterinarie \\ Universitá di Torino \\ Largo Paolo Braccini 2, 10095 Grugliasco (Italy) \\ Email: elisa.ciglieri, francesco.ferrini@unito.it \\ chiara.salio@unito.it
}

\begin{abstract}
Diabetic polyneuropathy is a major complication of diabetes mellitus, causing severe alterations of the neural circuits between spinal nerves and spinal cord. The analysis of 3D confocal images of dorsal root ganglia in diabetic mice, where different fluorescent markers are used to identify different types of nociceptors, can help understanding the unknown mechanisms of this pathology. Nevertheless, due to the inherent challenges of 3D confocal imaging, a thorough and comprehensive visual investigation is very difficult. In this work we introduce a tool, $3 D \mathrm{RG}$, that provides a fully-automated segmentation and 3D rendering of positively labeled nociceptors in a dorsal root ganglion, as well a quantitative characterisation of its immunopositivity to each fluorescent marker. Our preliminary experiments on 3D confocal images of entire dorsal root ganglia from healthy and diabetic mice provided very interesting insights about the effects of the pathology on two different types of nociceptors.
\end{abstract}

\section{INTRODUCTION}

D IABETIC polyneuropathy (DPN) is one of the most common and serious complications of diabetes mellitus, which includes several types of nerve damaging disorders [1]. High glycemic levels associated with diabetes create injuries to the small vessels supplying the nerves, with symptoms that can range from pain and numbness in the extremities to problems with the digestive system, urinary tract, blood vessels and heart. Such symptoms in minor cases can be extremely disabling and even fatal.

While literature had traditionally focused only on injures of the peripheral nerves (mainly legs and feet), early works have now unveiled possible implications of DPN at all levels of the nervous system, with special regards to the neural circuits between the spinal nerves and the spinal cord [2]. Most recent studies are especially focusing on the Dorsal Root Ganglia (DRGs), clusters of sensory neurons in the dorsal root of spinal nerves (see Figure 1), whose underlying mechanisms in relation to DPN are at the moment poorly understood. In particular, the role of nociceptive sensory cells in the DRGs (i.e. neurons specialised in conveying pain information to the higher centers) is now one of the main topics of investigation [3], [4]. The analysis of immunofluorescence images via 3D confocal microscopy has a major role in such investigations. In particular, entire DRGs of mice can be dissected out and stained with multiple fluorescent markers, each targeting a specific type of nociceptor. The result is a complex multi-coloured stack of images, where different nociceptors are labelled by fluorochromes emitting signal of a known spectral range, hence they can be imaged in separate color channels (see left part of Figure 2). Typically used markers include biotin-conjugated Isolectin B4 (IB4) and the antibody for Calcitonin Gene-Related Peptide (CGRP), which identify the unmyelinated non-peptidergic and the small peptidergic neurons in the DRGs, respectively [3].

While the imaging technology per se is widely acknowledged for being a valuable support to this type of study, the analysis of 3D images of DRGs remains a challenging task. First, because distinguishing the positively stained neural cells is made difficult by the presence of noise and artefacts (e.g. spurious fluorescence, black spots, etc.), which are intrinsic limitations of immunofluorescence. Second, because the 3D nature of the images makes manual analysis unfeasible. To the best of our knowledge, there is no availability of a completely automated tool able to support this type of analysis. Hence, the data presented by most of the published works in this context are obtained with semi-automated procedures,

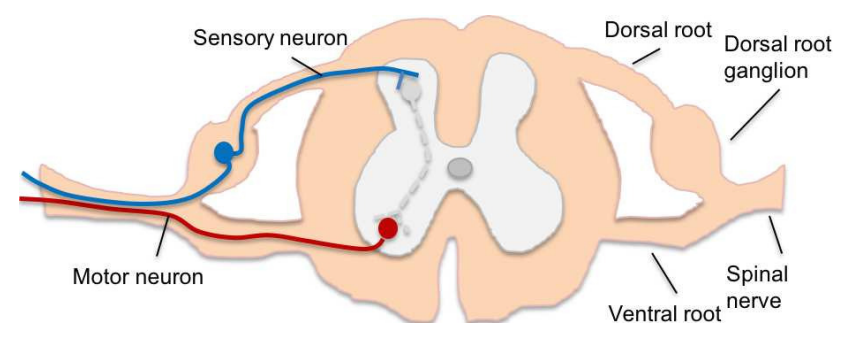

Figure 1. Cross-section of spinal cord. 


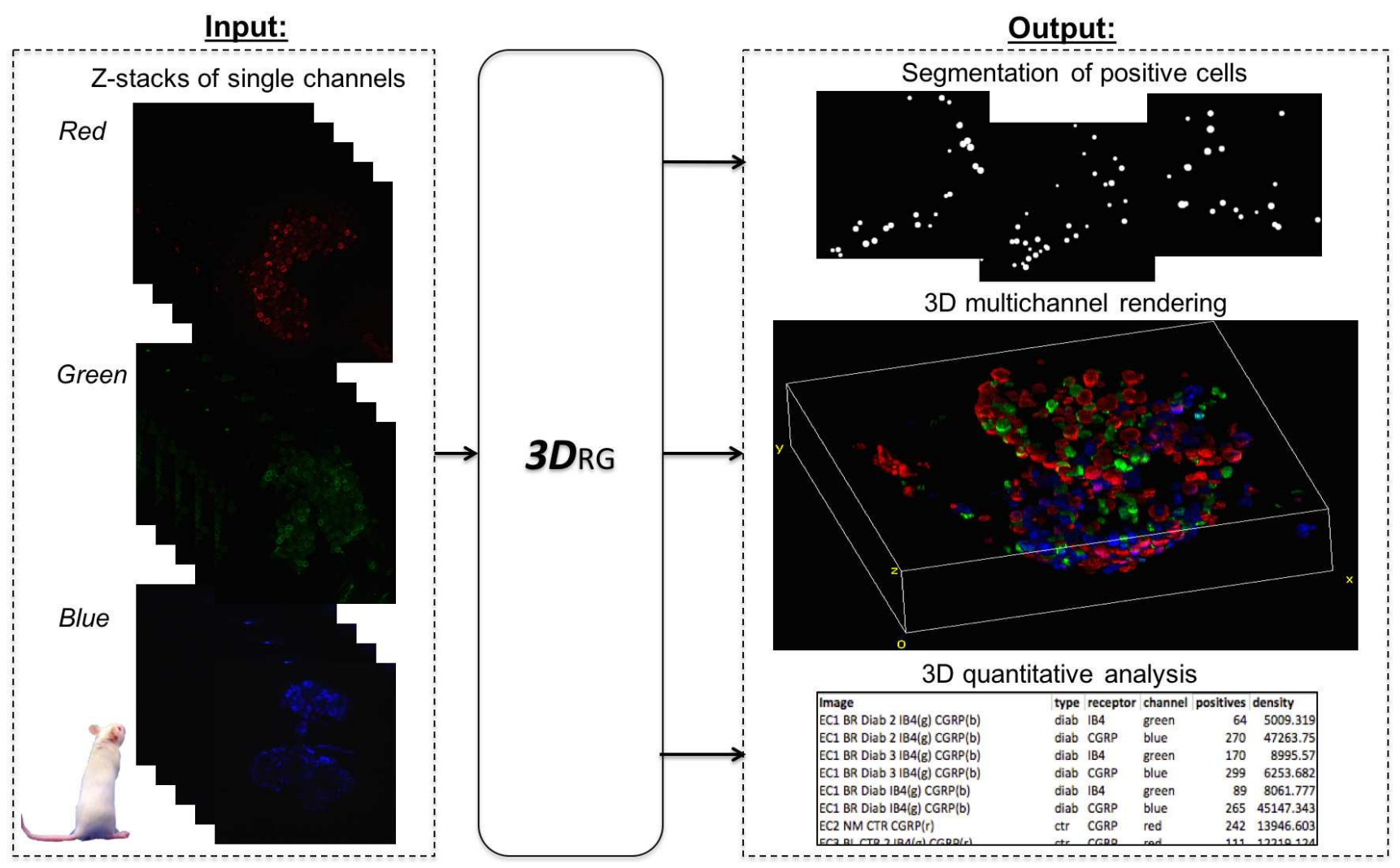

Figure 2. Simplified input/output diagram.

where the positive cells in few significant slices of the stack are distinguished from the background based on user-defined thresholds or standard 2D thresholding techniques. Then, the immunopositivity of the sample is quantified based on the number and average intensity of such cells [5], [3]. This process has two evident limitations: (i) it lacks reproducibility, due to the inherent subjectivity of user-interaction; (ii) by counting positive cells only on selected slices, it does take the 3D nature of the images into proper account. For example, a single neuron may span more consecutive slices of the stack, hence it should be counted only once.

As a solution to these problems, we propose $3 D \mathrm{RG}$, a tool for the immunofluorescence analysis of DRG samples. The main contribution of this work is two-fold. First, a fully automated 3D segmentation and 3D rendering of the positively labeled DRG neurons, based on a 3D spatial filtering technique. Second, a more accurate analysis of immunopositivity, which quantifies the number of positive cells and amount of fluorescent signal in the whole DRG stack.

Besides improving the feasibility and objectivity of DRG image analysis, 3DRG allows the analysis of the spatial relations between cells marked by different types of antibodies. This opens up new perspectives in the investigation of the role and interaction of different types of nociceptor in the context of DPN alterations.

This paper is structured as follows. In Section II we provide the technical details of the three modules of our proposed tool. In Section III we characterise the DRG samples used in our preliminary experiments. In Section IV we report and discuss the obtained results. Finally, in Section V we draw conclusions and provide future perspectives of our work.

\section{Proposed Method}

As reported in Figure 2, our proposed tool 3DRG receives as input the digitalised $\mathrm{z}$-confocal stacks of the immunolabelled DRG samples, as returned by the confocal microscope. Separate modules of the tool provide the following output:

1) Segmentation of positive cells.

2) $3 \mathrm{D}$ multichannel rendering.

3) $3 \mathrm{D}$ quantitative analysis.

In the following, we describe the three modules in detail.

\section{A. Segmentation of positive cells}

The immunolabelled DRG cells are automatically detected in the 3D volume, discarding noise, spurious objects and artefacts. This is obtained by a 3D cell segmentation technique whose main steps are reported in the flow-charts of Figure 3.

1) Preprocessing: First, some preprocessing is performed on the 2D slices of the stacks, in order to ease the segmentation process by generally improving the image quality. The contrast between cells and background is enhanced by a combination of Contrast Limited Adaptive Histogram Equalisation technique [6] and background subtraction, where a simple model 

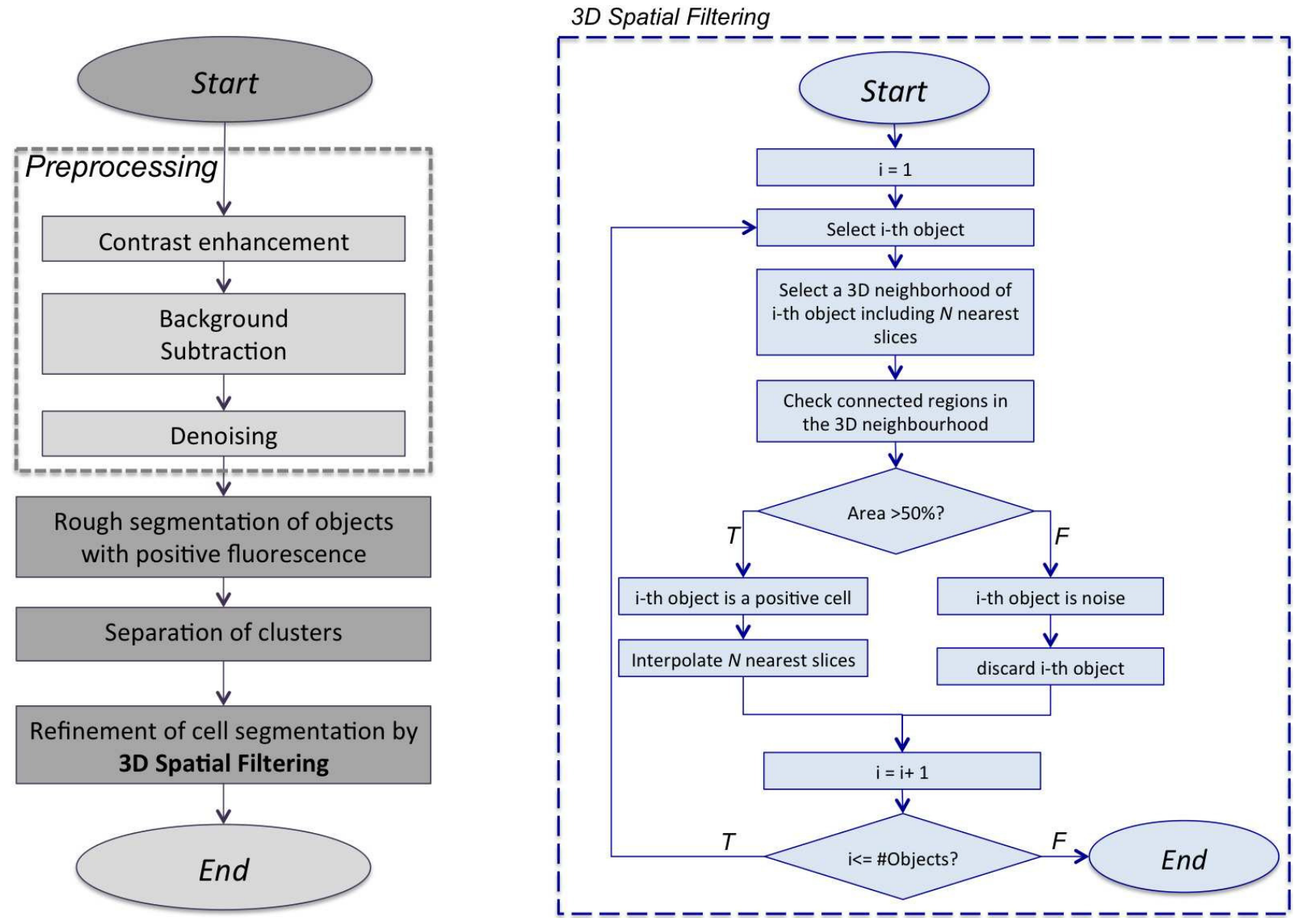

Figure 3. 3D Cell Segmentation: flow-chart.

of background computed by average filtering is subtracted to the original image. Following contrast enhancement, median filtering is performed to reduce high frequency noise preserving significant details of the image such as the borders of DRG cells.

2) Rough segmentation of objects with positive fluorescence: After preprocessing, fluorescent objects are roughly distinguished from the dark background by a 2D segmentation technique applied to each slice of the stack. This step implements a spatial fuzzy c-means algorithm (SFCM).

Standard fuzzy c-means (FCM) is a widely used clustering technique that partitions the data into a number of groups working towards the minimisation of the distance of points within the same cluster. The fuzziness of such process lies in assigning to the data points a $[0,1]$ membership level to each of the clusters (the so-called membership functions).

In order to decrease sensitivity to noise and reduce the spurious blobs, which are recurring problems in fluorescent image segmentation, 3DRG uses a variant of conventional FCM that incorporates local spatial information into its implementation, by summing up the membership functions in the neighbourhood of each pixel [7]. After clustering, the shapes of the foreground objects are regularised by means of standard morphological operations such as opening and holes filling.

3) Separation of clusters: The foreground regions returned by SFCM algorithm may either contain one individual cell or multiple touching cells, which need to be separated into individual objects. Based on the assumption that individual cells are approximately circular, 3DRG implements a Circle Hough transform (CHT), with the purpose of decomposing the input regions into a minimal number of circular components [8].

4) Refinement of cell segmentation: As ultimate step, the cell segmentation obtained by SFCM and CHT is refined by taking into account 3D spatial information (see 3D spatial filtering flow-chart in Figure 3). More specifically, per each foreground object, the filter generates a $3 \mathrm{D}$ neighbourhood by projecting such object onto $N$ consecutive slices of the z-stack (see Figure 4 ). $N$ is set to be roughly equal to the expected length of DRG cells. Hence, its value depends on slice thickness.

If the 3D neighbourhood does not contain a significant portion (at least $50 \%$ ) of positive regions, the corresponding object is interpreted as a spurious fluorescent spike, hence it is discarded. Otherwise, it is interpreted as part of a positive cell. In the latter case, the intensity values of consecutive slices in the $3 \mathrm{D}$ neighbourhood are interpolated along the z-axes, in 


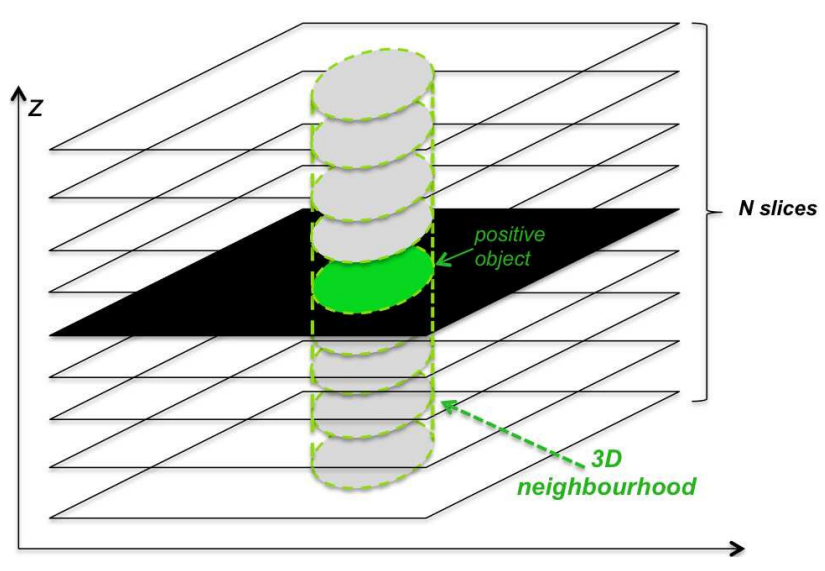

Figure 4. 3D neighbourhood for 3D spatial filtering.

order to remove black spots and ensure the 3D continuity of the cell.

More specifically, the pixels of the 3D neighbourhood in the slices above and below the positive object are replaced by the arithmetic mean of their corresponding pixels in the two closest slices, as follows:

$$
\operatorname{slice}(i)=\frac{\operatorname{slice}(i-1)+\operatorname{slice}(i+1)}{2},
$$

where $i$ is the $\mathrm{z}$-coordinate of the slice.

The final output of this step is a 3D volume where only the positive DRG cells have non-zero intensity values, proportional to the level of expression of the fluorescent marker.

\section{B. $3 D$ multichannel rendering}

3DRG generates a volume rendering of the DRG-positive stack as returned by the cell segmentation module, with the possibility of displaying one channel at a time or more channels together (as in the example of Figure 2).

The user can interactively rotate the volume and visualise ortho-slices (i.e. three orthogonal slices through the volume). This allows to analyse spatial relations between different markers (e.g. cell clusters, recurring patterns, etc.) that are otherwise impossible to appreciate.

\section{C. $3 D$ quantitative analysis}

The 3D sample is automatically characterized in terms of positive expression of each immunofluorescent marker.

This module allows the calculation of the following parameters:

- Number of positive cells $(P)$, as automatically identified by the $3 \mathrm{D}$ cell segmentation algorithm.

- 3D Density calculated as $P / V_{s}$, where $P$ is the number of positive cells and $V_{S}$ is the fraction of the sample volume with a non-zero fluorescent signal strength.

- Integrated Optical Density (IOD) calculated as the sum of mean intensity values $(M I V)$ of the foreground (i.e. the positive cells) in each slice of the volume. More specifically:

$$
I O D=\sum_{i=1}^{\# \text { Slices }} M I V_{i},
$$

where $M I V_{i}$ is computed as the sum of the intensities of the foreground pixels in $i-t h$ slice, divided by the total number of foreground pixels.

- Related Optical Density (ROD) calculated as:

$$
R O D=I O D_{F}-I O D_{B},
$$

where $I O D_{F}$ and $I O D_{B}$ are the $I O D$ values of the foreground and of the background, respectively.

\section{MATERIALS}

In this work we performed experiments on DRG samples belonging to two populations of mice, respectively diabetic and healthy subjects (here referred as controls). Such samples were obtained as follows.

Four weeks old CD-1 male mice were made diabetic after a single intraperitoneal injection of streptozotocin $(150 \mathrm{mg} / \mathrm{Kg})$, while controls received only the vehicle. Glucose levels of all the subjects were weekly monitored, in order to ensure the correct categorization of diabetics and controls. All animals were sacrificed at eight weeks of postnatal age. Then, DRGs were acutely excised and the connective tissue was dissolved by incubation in $5-10 \mathrm{mg} / \mathrm{mL}$ collagenase. The entire DRGs were then used for immunofluorescence.

DRGs were stained for two classical phenotypic markers of nociceptors, i.e. the calcitonin gene-related peptide (CGRP) and the isolectin B4 (IB4) by a rabbit antibody and a biotinconjugate, respectively. Whole DRGs z-stacks were then collected using confocal microscopy.

\section{PRELIMINARY RESUlts}

We run 3DRG on a total number of 90 DRG samples. Of the 90 samples, 45 belonged to diabetic subjects and 45 to controls. Hence, the two populations were perfectly balanced.

Positive DRG cells were automatically detected as explained in Section II-A, and volume rendering of the segmented cells was generated (see the example of Figure 5).

IB4 and CGRP markers were automatically quantified in all the 90 samples, as reported in Section II-C.

The obtained results are summarised by the box-plots of Figure 6, grouping the data into diabetic and control subjects, respectively.

On each box-plot, the central red line is the median value, and the box has edges corresponding to the 25th and 75th percentiles (the so-called inter-quartile range $I Q R$ ). The whiskers extend to the most extreme data points not considering outliers, while outliers are plotted individually using a red cross-shaped marker. As in previous works, all the values are normalised by the median of controls [3]. 

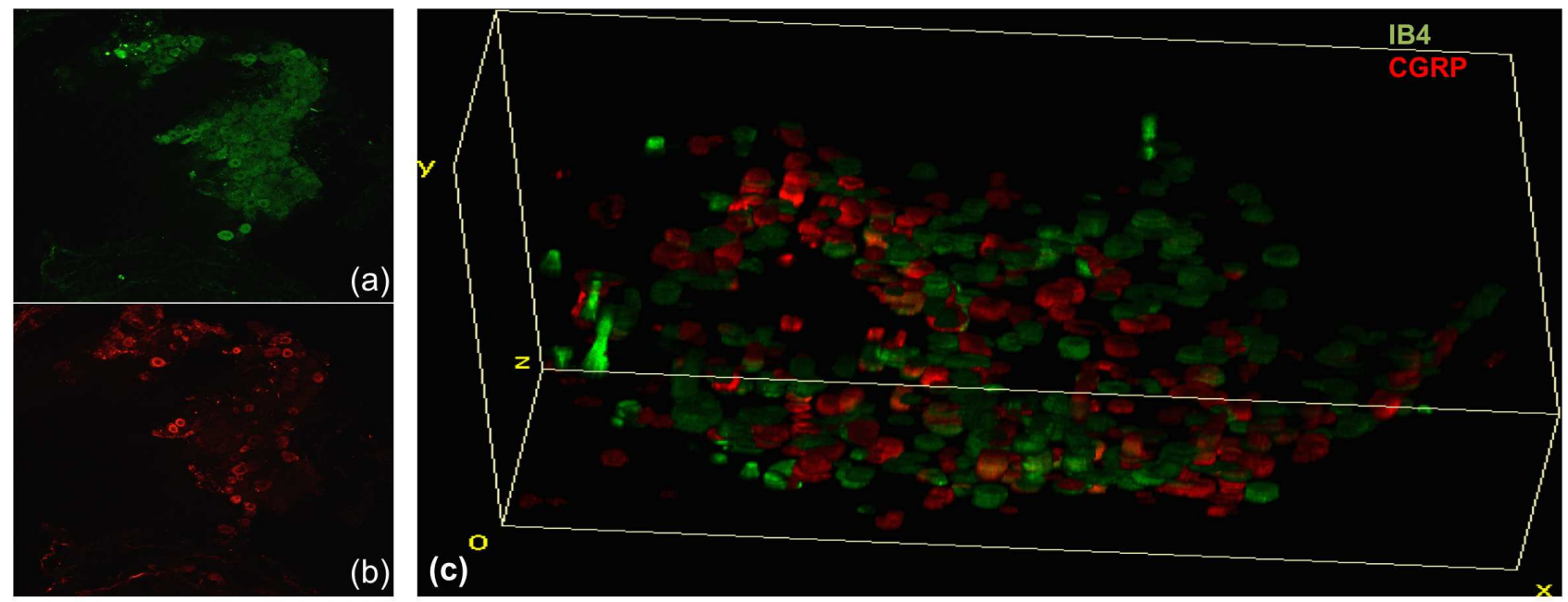

Figure 5. DRG sample. (a) IB4 labelling (green channel). (b) CGRP labelling (red channel). (c) Volume rendering of DRG positive cells automatically detected by $3 D$ RG tool.

In order to facilitate statistical comparisons between different groups, each box is displayed with a notch defining the confidence interval C.I. around the median, computed as:

$$
C I=\text { median value } \pm 1.57 \cdot \frac{I Q R}{\sqrt{N}}
$$

where $N$ is the number of observations and the constant 1.57 is an empirical value that is set to approximate a $95 \%$ confidence interval around the median [9].

Hence, when the notches of two groups of data points do not overlap, it interpreted as a strong evidence that the medians of the two samples are significantly different.

From the analysis of the box-plots in Figure 6, the following considerations can be drawn:

1) $3 \mathrm{D}$ quantitative analysis revealed relevant differences between control and diabetic groups.

In particular, the plots show a decrease of both IB4 and CGRP in the diabetic subjects.

2) the highest discrimination between controls and diabetics is obtained with IB4 marker. All four plots related to IB4 show non-overlapping notches between control and diabetic boxes, suggesting that the median values of the corresponding populations are different with a $95 \%$ confidence level.

The same happens with CGRP, but only when considering IOD and ROD values.

The experimental results automatically obtained with $3 D \mathrm{RG}$ are in line with the assumptions made by literature on neuroscience.

As reported by [3], [10], nonpeptidergic unmyelinated IB4labeled afferents may have a higher susceptibility to diabetes, and their decrease might be a reason for the early sensory dysfunctions associated with this pathology.

On the other hand, CGRP-labeled peptidergic fibers are also to a lesser extent involved in the deficit.

\section{Conclusions and Future Perspectives}

In this paper we presented an automated tool, $3 D \mathrm{RG}$, that is able to

1) perform a segmentation of positively labeled DRG neurons;

2) provide a multichannel $3 \mathrm{D}$ rendering of the labeled neurons;

3) characterise the immunopositivity of the sample to the DRG markers.

Our proposed tool allows to obtain better insights into the analysis of immunofluorescence DRG images applied to the study of diabetic neuropathies, for two main reasons.

First, differently from previous works, where counting of positive cells was performed in a semi-automated way and on only few slices of the $3 \mathrm{D}$ stack, $3 D \mathrm{RG}$ is able to characterise the sample in a fully-automated way, and by taking into account the whole 3D volume. This improves the repeatability and objectivity of the results, and allows to fasten and ease the analysis of large amount of image data.

Second, the $3 \mathrm{D}$ reconstruction and rendering of the segmented cells allows to visualise the 3D distribution of the different markers and to highlight spatial relations between different types of DRG afferents. This analysis cannot be performed on the original 3D stack due to noise and spurious fluorescence.

Results obtained in our preliminary experiments by running $3 D R G$ on DRG samples of healthy and diabetic mice were very interesting, in that they support the hypothesis that the alterations of the neural circuits between spinal nerve and spinal cord via the DRG might be involved in DPN, which is also confirmed by recent literature.

Indeed, fully-automated analysis of DRG images offers potential for huge improvements in the study of neural alterations related to diabetes. In our future work, we plan to extend $3 D R G$ to support a quantitative analysis of the $3 \mathrm{D}$ spatial 

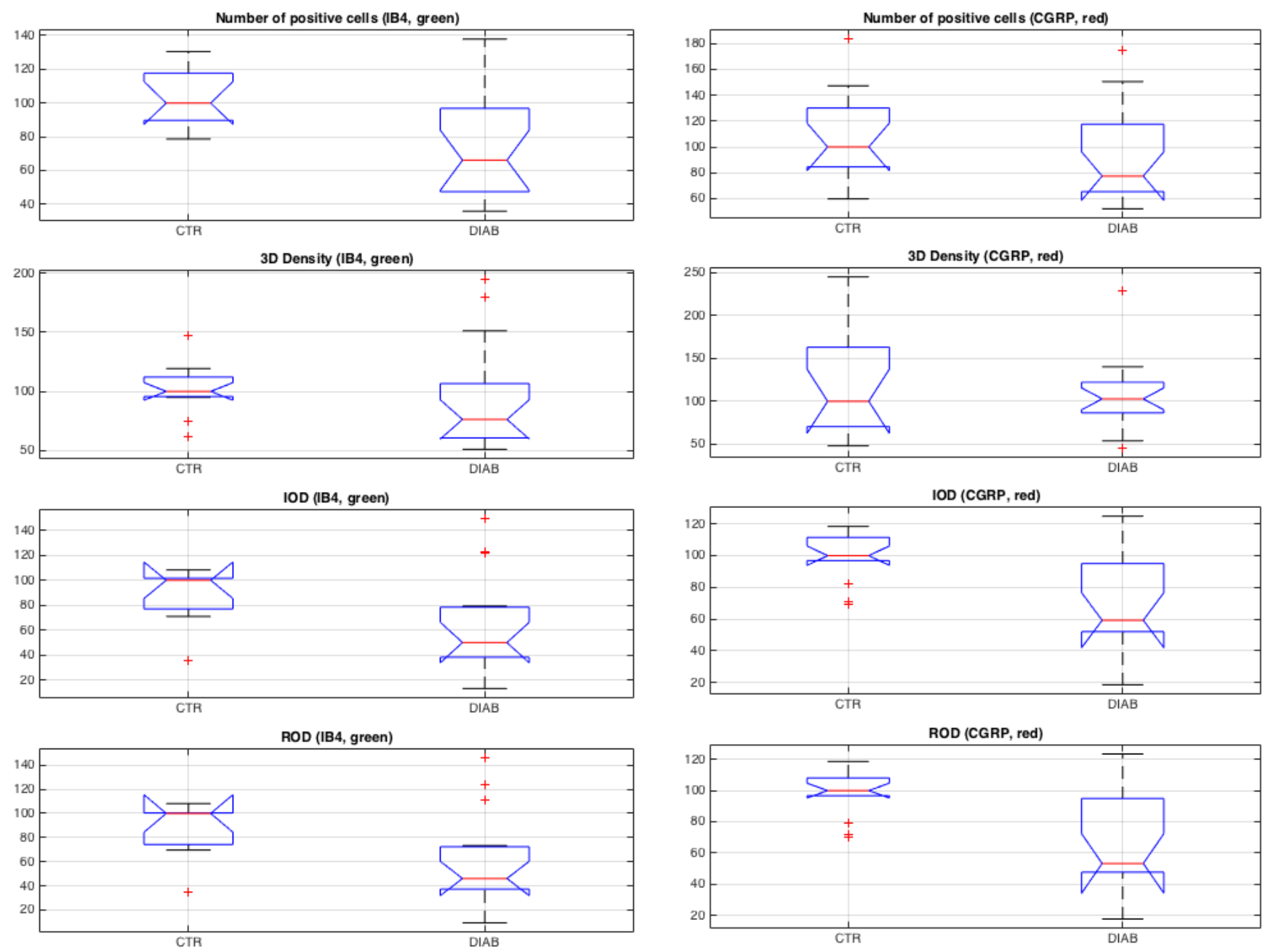

Figure 6. 3D quantitative immunofluorescence results on control and diabetic mice (values expressed as $\%$ of controls).

distribution of the different markers. This would allow to study the pathology-driven alterations of the relations between different DRG afferents, which is a type of analysis that was never performed before with immunofluorescence.

\section{REFERENCES}

[1] D. Ziegler and V. Fonseca, "From guideline to patient: a review of recent recommendations for pharmacotherapy of painful diabetic neuropathy," Journal of Diabetes and its Complications, vol. 29, no. 1 , pp. 146 - 156, 2015. doi: 10.1016/j.jdiacomp.2014.08.008. [Online]. Available: http://dx.doi.org/10.1016/j.jdiacomp.2014.08.008

[2] H. Kamiya, K. E. Weixian Zhang and, J. Wahren, and A. A. Sima, "Cpeptide reverses nociceptive neuropathy in type 1 diabetes," Diabetes, vol. 55, no. 12 , pp. 3581 - 3587, 2006. doi: 10.2337/db06-0396. [Online]. Available: http://dx.doi.org/10.2337/db06-0396

[3] Z.-Z. Kou, C.-Y. Li, J.-C. Hu, J.-B. Yin, D.-L. Zhang, Z.-Y. Wu, T. Ding, J. Qu, Y.-H. Liao, H. Li, and Y.-Q. Li, "Alterations in the neural circuits from peripheral afferents to the spinal cord: possible implications for diabetic polyneuropathy in streptozotocin-induced type 1 diabetic rats," Front Neur Circuits, vol. 8, no. 6, 2014. doi: 10.3389/fncir.2014.00006. [Online]. Available: http://dx.doi.org/10.3389/fncir.2014.00006

[4] E. S. Krames, "The role of the dorsal root ganglion in the development of neuropathic pain," Pain Medicine, vol. 15, no. 10, pp. 1669-1685, 2014. doi: 10.1111/pme.12413. [Online]. Available: http://dx.doi.org/10.1111/pme.12413
[5] R. Wang, A. Rossomando, D. W. Sah, M. H. Ossipov, T. King, and F. Porreca, "Artemin induced functional recovery and reinnervation after partial nerve injury," Pain, vol. 155, no. 3, pp. $476-$ 484, 2014. doi: 10.1016/j.pain.2013.11.007. [Online]. Available: http://dx.doi.org/10.1016/j.pain.2013.11.007

[6] K. Zuiderveld, "Contrast limited adaptive histogram equalization," in Graphics Gems IV. Academic Press Professional, Inc., 1994, pp. 474-485. [Online]. Available: http://dl.acm.org/citation.cfm?id=180895. 180940

[7] K.-S. Chuang, H.-L. Tzeng, S. Chen, J. Wu, and T.-J. Chen, "Fuzzy c-means clustering with spatial information for image segmentation," Computerized Medical Imaging and Graphics, vol. 30, no. 1, pp. 9 - 15, 2006. doi: 10.1016/j.compmedimag.2005.10.001. [Online]. Available: http://dx.doi.org/10.1016/j.compmedimag.2005.10.001

[8] T. Atherton and D. Kerbyson, "Size invariant circle detection," Image and Vision Computing, vol. 17, no. 11, pp. 795 - 803, 1999. doi: 10.1016/S0262-8856(98)00160-7. [Online]. Available: http: //dx.doi.org/10.1016/S0262-8856(98)00160-7

[9] J. Chambers, W. Cleveland, B. Kleiner, and P. Tukey, "Graphical Methods for Data Analysis," The Wadsworth Statistics/Probability Series. Boston, MA: Duxury, 1983. doi: 10.2307/2531418. [Online]. Available: http://dx.doi.org/10.2307/2531418

[10] S. Akkina, C. Patterson, and D. Wright, " $\{$ GDNF $\}$ rescues nonpeptidergic unmyelinated primary afferents in streptozotocintreated diabetic mice," Experimental Neurology, vol. 167, no. 1, pp. 173 - 182, 2001. doi: 10.1006/exnr.2000.7547. [Online]. Available: http://dx.doi.org/10.1006/exnr.2000.7547 